\title{
Demonstration of a Full-Duplex Bidirectional Spectrally Interleaved OCDMA/DWDM System
}

\author{
P. C. Teh, Student Member, IEEE, M. Ibsen, and D. J. Richardson
}

\begin{abstract}
We report on a full-duplex bidirectional spectrally interleaved optical code-division multiple access/dense wavelength-division-multiplexing demonstration based on 16-b $20-\mathrm{Gb} / \mathrm{s}$ quarternary phase coding gratings. Eight $622-\mathrm{Mb} / \mathrm{s}$ coded channels are transmitted in each direction and error-free performance obtained over a transmission distance of $44 \mathrm{~km}$ of nonzero dispersion shifted fiber.
\end{abstract}

Index Terms-Bragg grating, fiber-optic communication, matched filters, optical code-division multiplexing.

\section{INTRODUCTION}

$\mathbf{T}$ HERE IS AN emerging requirement for high-speed access networks capable of providing various forms of data service on demand to individual homes and businesses. Such networks require broad-band access techniques capable of supporting the required fine-channel granularity and bursty nature of data services. Optical code-division multiple access (OCDMA) is one such technology that has the potential to support a large number of asynchronous users with simple network topologies. OCDMA is a spread-spectrum broadcast technique in which each user of the network is allocated a unique optical code (address) [1]. This code can be used to label data bits broadcast onto the network that are intended for receipt by a particular user, or alternatively to label bits that have been sent from a particular user. This approach also provides a high level of security since information to be transmitted is optically encoded in the physical layer of the network.

Recently, superstructured fiber Bragg grating (SSFBG) technology has been shown to provide an attractive and highly flexible route to produce high performance and potentially low-cost optical code generation and recognition components as required for direct sequence (DS)-OCDMA systems [2]. We have previously demonstrated a unidirectional 16-channel OCDMA/wavelength-division-multiplexing (WDM) system based on SSFBGs using practical distributed feedback (DFB) fiber laser transmitters [3]. A typical access network would require data to be transmitted in both upstream and downstream directions (bidirectional) simultaneously (full-duplex) with no requirement for timing synchronization between channels. In this letter, we report on an eight-channel full-duplex bidirectional spectrally interleaved OCDMA/dense WDM (DWDM) access network based on four-level phase encoded SSFBGs.

Manuscript received September 6, 2002; revised November 21, 2002.

P. C. Teh is with the Optoelectronics Research Centre, University of Southampton, Southampton SO17 1BJ, U.K. (e-mail: pct@orc.soton.ac.uk).

M. Ibsen and D. J. Richardson are with the Optoelectronics Research Centre, University of Southampton, Southampton SO17 1BJ, U.K. and also with

Southampton Photonics Inc., Southampton, U.K.

Digital Object Identifier 10.1109/LPT.2002.807912
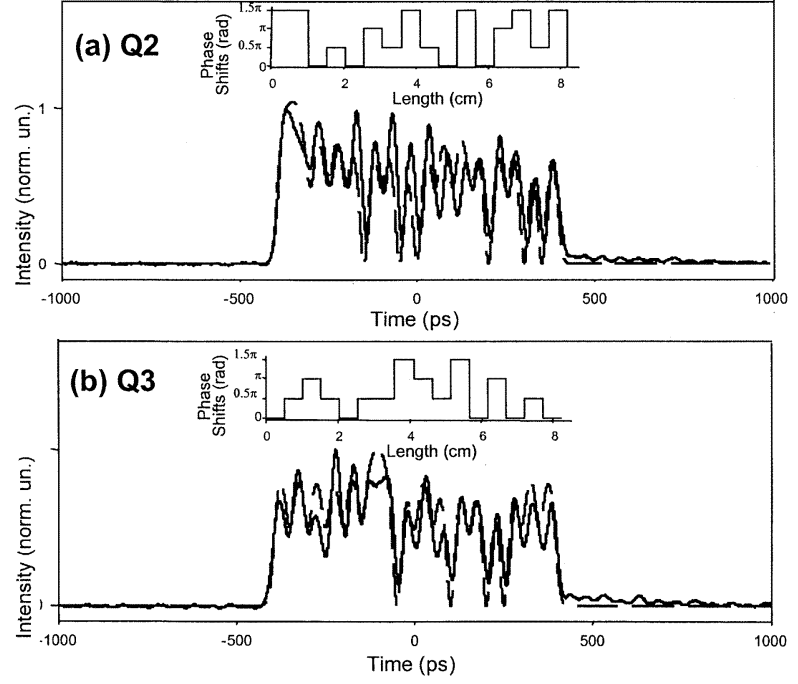

Fig. 1. Oscilloscope traces (measured-solid line, and calculated-dashed line) of the (a) encoded waveform after reflection from SSFBG Q2 and (b) encoded waveform after reflection from SSFBG Q3. The measured resolution was $\sim 20 \mathrm{ps}$. Inset shows the four-level phase modulation profile imposed upon the grating during the writing process.

In this demonstration, the upstream channels are spectrally interleaved between adjacent downstream channels in order to make efficient use of the fiber bandwidth. Most importantly, we demonstrate the application of the inherent wavelength selectivity of the coding SSFBGs to perform DWDM filtering at both OCDMA encoders and decoders eliminating the need for additional filtering components.

\section{GRATINGS FABRICATION AND CHARACTERIZATION}

An SSFBG has a rapidly varying refractive index modulation (of uniform amplitude and pitch), onto which an additional slowly varying amplitude/phase CDMA code (superstructure) has been imposed along its length. For a weak SSFBG (reflectivity $R<20 \%$ ) the shape of the impulse response follows directly the shape of the spatial superstructure. Short pulses reflected from the grating are thus reshaped into coded pulse sequences with the same form as the superstructure profile used to write the grating. Pattern recognition can then be obtained by matched filtering the resulting coded signal using a decoder grating with the conjugate impulse response (i.e., a grating with a spatially reversed superstructure profile relative to that of the encoder grating [2]).

A total of 16 different coding SSFBGs (four OCDMA codes centered on four DWDM wavelengths) were fabricated. The gratings have a chip duration of $50 \mathrm{ps}$, resulting in a total grating length of $8.22 \mathrm{~cm}$. The peak reflectivity of all of these gratings 


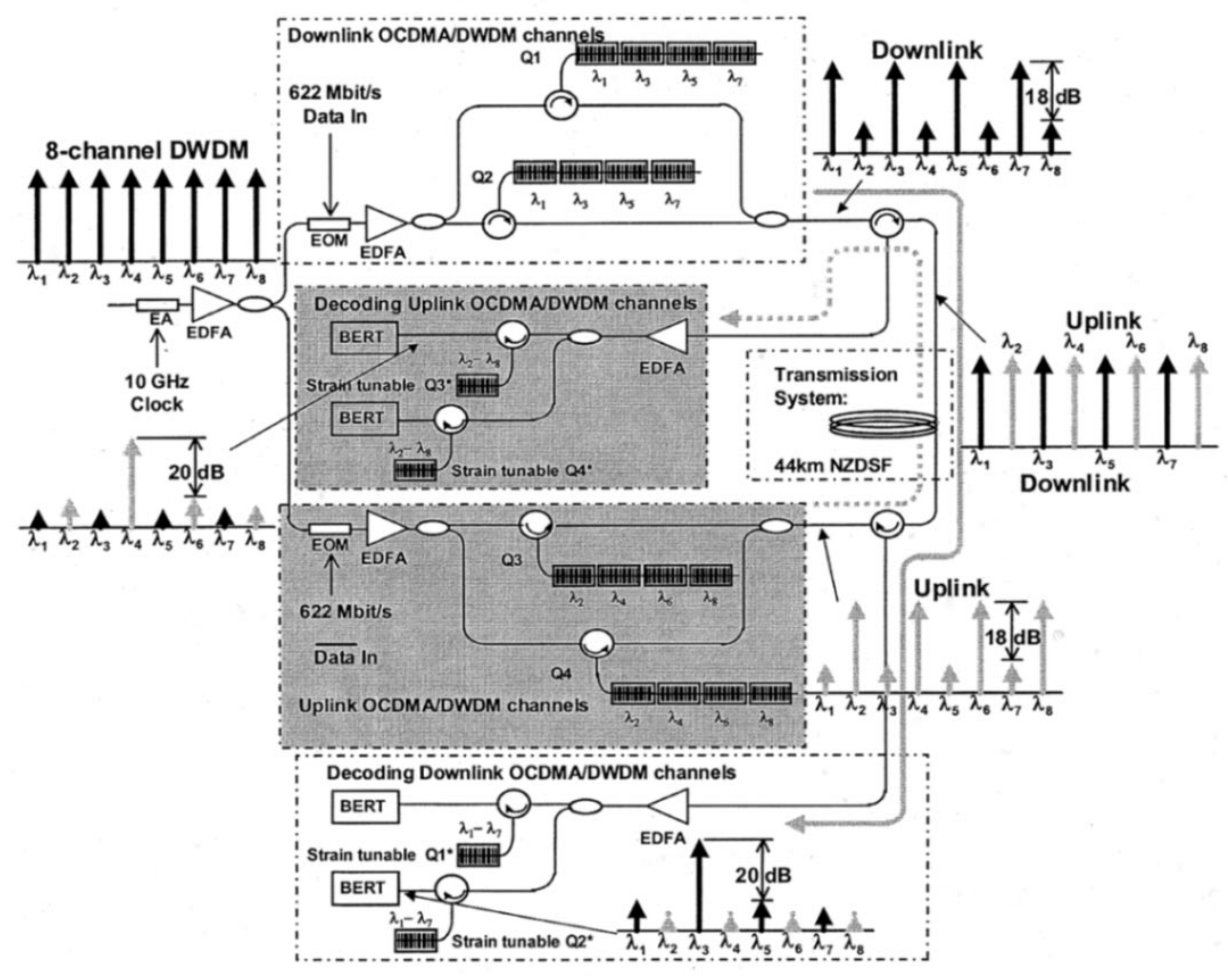

Fig. 2. Full-duplex bidirectional spectrally interleaved OCDMA/DWDM experimental setup. Q1-Q4: SSFBGs matched to the DWDM wavelengths. The schematic shows the DWDM wavelength filtering using SSFBGs at various points within the system.

is $\sim 25 \%$. The gratings were fabricated using our continuous grating writing technique requiring only a single uniform-pitch phase mask [4], [5]. This technique has the accuracy to allow for excellent control of the amplitude and phase of the refractive index modulation along the grating length. The four-level phase shift keyed OCDM codes are derived from the Family A sequences and which provide for more desirable auto/cross correlation characteristics than lower level coding schemes such as unipolar and bipolar code sequences [6].

In Fig. 1(a) and (b), we show the oscilloscope traces of the Q2 and Q3 coded channels obtained by reflecting 20-ps pulses from gratings Q2 and Q3, respectively. As expected, the gratings spread the incident 20-ps pulses over a time period of $\sim 800$ ps. The phase modulation profile of codes Q2 and Q3 imposed upon the gratings during the writing process are shown inset. The good agreement between the experimental data and the theoretical predictions highlight the quality of the SSFBGs fabricated.

\section{EXPERIMENTAL RESULTS}

The experimental setup used to demonstrate the bidirectional OCDMA/DWDM network is shown in Fig. 2. Eight continuous-wave $(\mathrm{CW})$ lasers separated in frequency by $50 \mathrm{GHz}$ are used to generate eight DWDM channels (denoted by $\lambda_{1}-\lambda_{8}$ ). The combined DWDM channels are directed into an electroabsorption (EA) modulator that was driven with a $10-\mathrm{GHz}$ sinusoidal signal in order to produce 10-GHz 20-ps DWDM pulse trains. These pulse trains were then split using a fiber coupler to generate the upstream and downstream links. The downstream DWDM pulse trains were gated down to $622 \mathrm{MHz}$ and modulated with a $2^{7}-1$ pseudorandom data sequence using a $\mathrm{LiNbO}_{3}$ intensity modulator so as to generate 20-ps
$622-\mathrm{Mb} / \mathrm{s}$ data streams. These modulated pulse trains were reflected from an array of eight coding gratings; two different OCDM codes (Q1 and Q2) centered on odd wavelengths, denoted by $\lambda_{1}, \lambda_{3}, \lambda_{5}$, and $\lambda_{7}$ so as to generate eight separate coded data channels $(2 \mathrm{OCDM} \times 4$ DWDM on a $100-\mathrm{GHz}$ grid). Note that in our demonstration no additional wavelength filtering components are required to separate the individual 50-GHz DWDM signals before reflection onto each SSFBG since the SSFBG is capable of performing simultaneous code generation and DWDM wavelength selection with $\sim 18 \mathrm{~dB}$ of extinction between adjacent 50-GHz DWDM wavelengths (see Fig. 2). All eight coded channels generated from the array of SSFBGs were combined and then transmitted over a distance of $44 \mathrm{~km}$ in nonzero dispersion-shifted fiber (NZDSF) having a total dispersion of $118.8 \mathrm{ps} / \mathrm{nm}$. The transmitted data streams were then split using another fiber coupler and fed onto two decode gratings matched to the particular codes and wavelengths of interest.

Similarly, the other output (upstream) was gated and modulated with the complementary data at the same data rate before being reflected from another array of eight coding gratings comprising two OCDM codes (Q3 and Q4) centered on even wavelengths, denoted by $\lambda_{2}, \lambda_{4}, \lambda_{6}$, and $\lambda_{8}$ for upstream link. The eight coded upstream signals were then combined and transmitted from the opposite end of the same NZDSF. Again, two decode gratings were included at the receiving end, matched to the particular codes and wavelengths of interest. In this implementation, the upstream coded channels are spectrally interleaved between the adjacent $100-\mathrm{GHz}$ downstream coded channels as illustrated in Fig. 2. The performance of both upstream and downstream pairs of OCDM coded channels can be simultaneously analyzed by measuring the bit-error-rate 

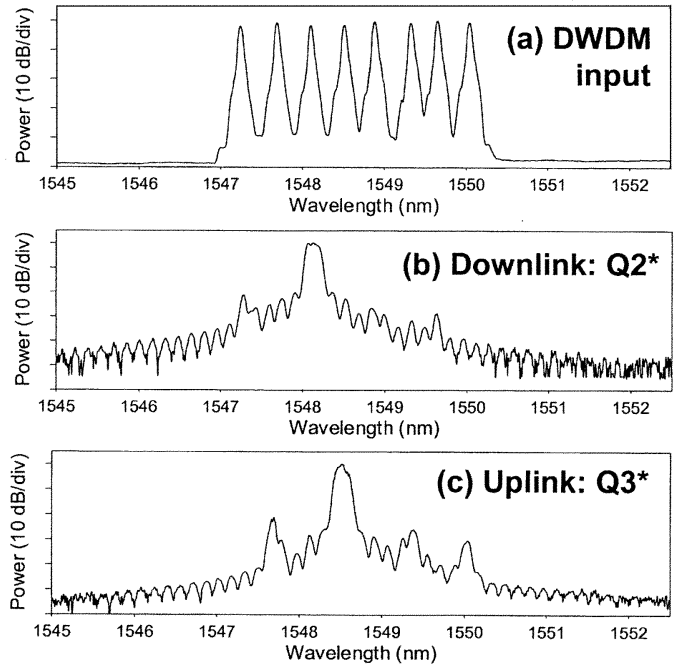

Fig. 3. (a) Combined eight DWDM wavelengths separated in frequency by $50 \mathrm{GHz}$. (b) Downstream optical spectra obtained after matched filtering with decode grating Q2*. (c) Similar upstream results after matched filtering with grating Q3*.

(BER) performance of both the upstream and downstream coded channels.

The optical spectrum of the eight DWDM wavelengths separated in frequency by $50 \mathrm{GHz}$ is shown in Fig. 3(a). Fig. 3(b) shows the optical spectrum of the downstream decoded response after matched filtering using grating Q2*. For convenience, we denote the individual matched filters to the individual codes using the notation $\mathrm{Q} 2 *, \mathrm{Q} 3 *$ where for example $\mathrm{Q} 2 *$ is the matched decoder to grating Q2. An extinction ratio of $\sim 20 \mathrm{~dB}$ between adjacent $100-\mathrm{GHz}$ OCDMA wavelength channels is obtained. Similar extinction ratios are observed for the upstream decoded response after matched filtering using grating Q $3^{*}$ as shown in Fig. 3(c). The above optical spectra show that the inherent wavelength selectivity of the SSFBG can be used both to provide DWDM wavelength drop function as well as the OCDMA decoding function for the in-band signals, eliminating the requirement for additional wavelength channel filtering elements at the receiver.

The performance of the full-duplex OCDMA/DWDM bidirectional system was characterized by measuring the BER of both upstream and downstream channels versus the total optical power at the receiver. These results are plotted in Fig. 4. Error-free performance is obtained for all the measured channels with $\sim 3-\mathrm{dB}$ power penalty when compared to the laser back-to-back measurement for both upstream and downstream transmission. This power penalty can be eliminated with the incorporation of dispersion-compensating elements, albeit with an increase in system complexity. Most importantly, almost no power penalty is observed when comparing both upstream and downstream channel performances. (Any variation in the comparison between upstream and downstream power penalty we believe is due to slight variation in the quality of the code: decode gratings used in the experiment.) If some sort of nonlinear thresholding is introduced at the receiver, we should anticipate a significant improvement in both system performance and capacity both in terms of number of simultaneous users per wavelength and data rate per OCDM channel

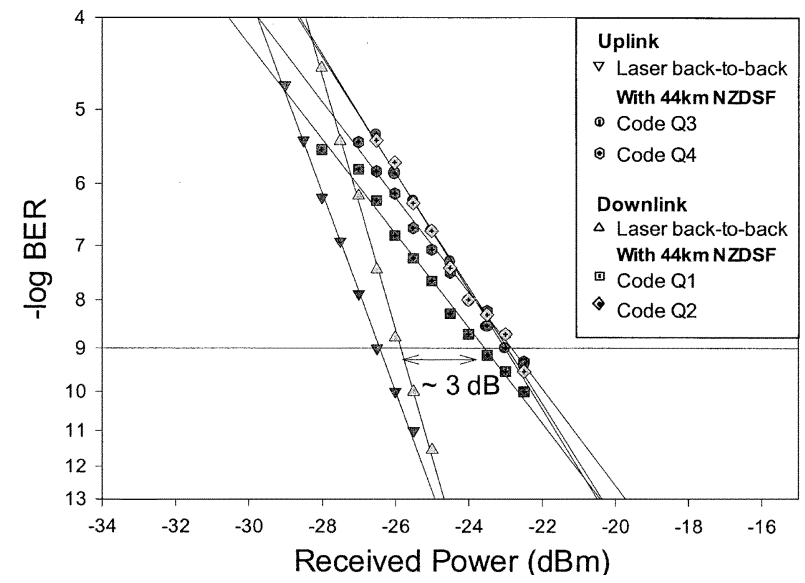

Fig. 4. BER measurements for bidirectional OCDMA/DWDM experiment at a data rate of $622 \mathrm{Mb} / \mathrm{s}$.

[7]. Again though, there would be a cost in terms of system complexity and practicality.

\section{CONCLUSION}

We have experimentally demonstrated a simple SSFBG coding-decoding and wavelength-selection approach in a bidirectional spectrally interleaved OCDMA/DWDM system. While fixed phase-code encoder and decoder SSFBGs were used in these experiments, the implementation of simple dynamically code-tunable encoder SSFBGs based on uniform FBG [8] would enhance the flexibility and reconfigurability of such access networks. These results further demonstrate that the SSFBG approach provides an extremely powerful and flexible way to perform many of the elementary optical processing functions required within both OCDMA and packet switched networks.

\section{REFERENCES}

[1] N. Karafolas and D. Uttamchandani, "Optical fiber code division multiple access networks: A review," Opt. Fiber Technol., vol. 2, pp. 149-168, 1996

[2] P. C. Teh, P. Petropoulos, M. Ibsen, and D. J. Richardson, “A comparative study of the performance of 7 and 63-chip optical code division multiple access encoders and decoders based on superstructure fiber Bragg gratings," J. Lightwave Technol., vol. 19, pp. 1352-1365, Sept. 2001.

[3] P. C. Teh, M. Ibsen, L. B. Fu, J. H. Lee, Z. Yusoff, and D. J. Richardson, "A 16-channel OCDMA system (4 OCDMA $\times 4$ WDM) based on 16-chip, $20 \mathrm{Gchip} / \mathrm{s}$ superstructure fiber Bragg gratings and DFB fiber laser transmitters," presented at the Proc. OFC'2002, Anaheim, CA, 2002, Invited Paper ThEE1.

[4] M. Ibsen, M. K. Durkin, M. J. Cole, and R. I. Laming, "Sinc-sampled fiber Bragg gratings for identical multiple wavelength operation," IEEE Photon. Technol. Lett., vol. 10, pp. 842-844, June 1998.

[5] M. Ibsen, M. K. Durkin, M. J. Cole, M. N. Zervas, and R. I. Laming, "Recent advances in long dispersion compensating fiber Bragg gratings," IEE Publications, vol. 0963-3308-ref., no. 1999/023, 1999.

[6] S. Boztas, R. Hammons, and P. V. Kumar, "4-phase sequences with nearoptimum correlation properties," IEEE Trans. Inform. Theory, vol. 38, pp. 1101-1113, May 1992.

[7] J. H. Lee, P. C. Teh, P. Petropoulos, M. Ibsen, and D. J. Richardson, "Reduction of interchannel interference noise in a two-channel grating based OCDMA system using a nonlinear optical loop mirror," IEEE Photon. Technol. Lett., vol. 13, pp. 529-531, May 2001.

[8] M. R. Mokhtar, M. Ibsen, D. J. Richardson, and M. Ibsen, "16-bit multilevel reconfigurable phase encoder for all optical header generation/recognition based on a uniform fiber Bragg grating," in Proc. 7th OECC'2002, Yokohama, Japan, 2002, Paper PD-2-2. 